International Journal of Electrical and Computer Engineering (IJECE)

Vol. 11, No. 1, February 2021, pp. 628 635

ISSN: 2088-8708, DOI: 10.11591/ijece.v11i1.pp628-635

$\square \quad 628$

\title{
Conformable Chebyshev differential equation of first kind
}

\author{
Abedallah Rababah \\ Department of Mathematical Sciences, United Arab Emirates University, United Arab Emirates \\ Department of Mathematics, Jordan University of Science and Technology, Jordan
}

\begin{tabular}{|c|c|}
\hline Article Info & ABSTRACT \\
\hline Article history: & In this paper, the Chebyshev-I conformable differential equation is considered. \\
\hline $\begin{array}{l}\text { Received Oct 31, } 2019 \\
\text { Revised Jun 20, } 2020 \\
\text { Accepted Jul 1,2020 }\end{array}$ & $\begin{array}{l}\text { A proper power series is examined; there are two solutions, the even solution and } \\
\text { the odd solution. The Rodrigues' type formula is also allocated for the conformable } \\
\text { Chebyshev-I polynomials. }\end{array}$ \\
\hline
\end{tabular}

\section{Keywords:}

Chebyshev-I differential equation

Chebyshev-I polynomials

Conformable differentiation

Rodrigues' formula

This is an open access article under the CC BY-SA license.

\section{Corresponding Author:}

Abedallah Rababah,

Department of Mathematical Sciences,

United Arab Emirates University, 15551 Al Ain, United Arab Emirates.

E-mail: rababah@uaeu.ac.ae

\section{INTRODUCTION}

It is well known that the year 1695 is considered as the birthday of the so called field of fractional calculus. L'Hospital wrote a letter on September 30, 1695 to Leibniz wondering about the notation used in the publications for the derivative of the function

$$
f(x)=x, \frac{d^{n} f}{d x^{n}}
$$

L'Hospital asked Leibniz the following question: what would the result be if $n=\frac{1}{2}$. Leibniz wrote back to L'Hospital: an apparent paradox, from which one day useful consequences will be drawn. Since then, many Mathematicians tried their definitions for a fractional order derivative; most of these definitions are based on integration formulas. The commonly used definitions are those given by Riemann-Liouville, Caputo, and Grünwald-Letnikov of non-integer derivatives. These definitions are summarized in the following lines [1-5]. Let $n$ be a positive integer, $\alpha \in(n-1, n]$, and $x \geq a$.

- Riemann-Liouville (left-sided) $\alpha$ fractional derivative of $f$ is defined by:

$$
D_{a}^{\alpha}[f(x)]=\frac{1}{\Gamma(n-\alpha)} \frac{d^{n}}{d x^{n}} \int_{a}^{x}(x-\xi)^{n-1-\alpha} f(\xi) d \xi
$$

where $\Gamma(x)$ is the well known Gamma function.

- Grünwald-Letnikov (left-sided) $\alpha$ fractional derivative of $f$ is defined by:

$$
D_{a}^{\alpha}[f(x)]=\lim _{h \rightarrow 0} \frac{1}{h^{\alpha}} \sum_{k=0}^{[n]}(-1)^{k} \frac{\Gamma(\alpha+1) f(x-k h)}{\Gamma(k+1) \Gamma(\alpha-k+1)} .
$$


- Caputo (left-sided) derivative $\alpha$ fractional derivative of $f$ is defined by:

$$
D_{a}^{\alpha}[f(x)]=\frac{1}{\Gamma(n-\alpha)} \int_{a}^{x}(x-\xi)^{n-1-\alpha} \frac{d^{n}}{d \xi^{n}}[f(\xi)] d \xi ., \quad x \geq a .
$$

For a complete list of fractional derivatives, see the survey in [2].

An acceptable definition for fractional derivatives should agree with the ordinary derivatives. As noticed in [3], the only property inherited by all definitions of fractional derivatives is the linearity property; they counted the following drawbacks of one definition or another: the Riemann-Liouville derivative does not reproduce the derivative of a constant to be 0 . All definitions do not satisfy the product, quotient, and chain rules. There are other drawbacks for the existing definitions of fractional derivatives.

The well-known Chebyshev differential equation of first kind is given in the form [6-13]:

$$
\left(1-x^{2}\right) y^{\prime \prime}-x y^{\prime}+n^{2} y=0, \quad n \in N .
$$

Expanding the solution using a power series around the ordinary point $x=0$ yields the well-known Chebyshev polynomials of first kind, $T_{n}(x)$. For Multivariate case, see [14-16]. The Chebyshev-I polynomials fulfill the following orthogonality conditions $[6,7]$ :

$$
\int_{-1}^{1} \frac{T_{n}(x) T_{m}(x)}{\sqrt{1-x^{2}}} d x=0, \text { whenever } n \neq m .
$$

\section{RESEARCH METHOD}

In this section, the conformable Chebyshev equation of first kind is defined and solved to give the conformable Chebyshev functions of first kind. For $\alpha \in(0,1]$, the $\alpha$ conformable derivative of $y$ is denoted by $D^{\alpha} y$; the second $\alpha$ conformable derivative is denoted by $D^{\alpha} D^{\alpha} y$. Recently, in [3] the conformable derivative is given in the following definition, see also [4].

Definition Let $f:[0, \infty) \mapsto R$, then the conformable derivative of $f$ of order $\alpha$ is defined by:

$$
D^{\alpha} f(x)=\lim _{\epsilon \rightarrow 0} \frac{f\left(x+\epsilon x^{1-\alpha}\right)-f(x)}{\epsilon}, x>0, \alpha \in(0,1) .
$$

If $f$ is $\alpha$ differentiable in $(0, a), a>0$, and

$$
\lim _{x \rightarrow 0^{+}} D^{\alpha} f(x)
$$

exists, then we set

$$
D^{\alpha} f(0)=\lim _{x \rightarrow 0^{+}} D^{\alpha} f(x) .
$$

Let $a, b, c, p \in R$ and $f$ and $g$ be $\alpha$ differentiable at a point $x>0$, then the conformable derivative satisfies the following properties:

$$
\begin{array}{r}
D^{\alpha}(c)=0, \\
D^{\alpha}\left(x^{p}\right)=p x^{p-\alpha}, \\
D^{\alpha}(a f+b g)=a D^{\alpha} f+b D^{\alpha} g .
\end{array}
$$

It is satisfactory to consider the $\alpha$ conformable derivative for $\alpha \in(0,1]$. If $\alpha \in(n, n+1], n \in N$, then the conformable derivative, if it exist, is defined by

$$
D^{\alpha} f(x)=D^{n}\left(D^{\alpha-n} f(x)\right) .
$$

A fractional power series of $\alpha$ is defined as a series of the form

$$
\sum_{k=0}^{\infty} a_{k} x^{k \alpha}, \alpha \in(0,1]
$$


Throughout this paper, let $\mathcal{P}_{n}$ be the set of all polynomials of degree $\leq n$.

For $\alpha \in(0,1]$, we define the conformable Chebyshev differential equation of the first kind by:

$$
\left(1-x^{2 \alpha}\right) D^{\alpha} D^{\alpha} y-\alpha x^{\alpha} D^{\alpha} y+\alpha^{2} n^{2} y=0, \quad n \in N .
$$

For few applications using the Chebyshev polynomials, see [17-26]. A solution of (5) in a proper power series of $\alpha$ is considered. Let this solution be given by a conformable power series of $\alpha$ as

$$
y=\sum_{k=0}^{\infty} a_{k} x^{k \alpha}
$$

\section{RESULTS AND ANALYSIS} given by:

Using the rules of conformable derivatives (3), then the $\alpha$ first and second conformable derivatives are

$$
\begin{array}{r}
D^{\alpha} y=\sum_{k=1}^{\infty} \alpha k a_{k} x^{(k-1) \alpha}, \\
D^{\alpha} D^{\alpha} y=\sum_{k=2}^{\infty} \alpha^{2} k(k-1) a_{k} x^{(k-2) \alpha} .
\end{array}
$$

tion:

Substituting the proper power series and its conformable derivatives in (5) yields the following equa-

$$
\left(1-x^{2 \alpha}\right) \sum_{k=2}^{\infty} \alpha^{2} k(k-1) a_{k} x^{(k-2) \alpha}-\alpha x^{\alpha} \sum_{k=1}^{\infty} \alpha k a_{k} x^{(k-1) \alpha}+\alpha^{2} n^{2} \sum_{k=0}^{\infty} a_{k} x^{k \alpha}=0 .
$$

This is further simplified to

$$
\begin{gathered}
\sum_{k=2}^{\infty} \alpha^{2} k(k-1) a_{k} x^{(k-2) \alpha}-\sum_{k=2}^{\infty} \alpha^{2} k(k-1) a_{k} x^{k \alpha}-\alpha x^{\alpha} \sum_{k=1}^{\infty} \alpha k a_{k} x^{(k-1) \alpha}+ \\
\alpha^{2} n^{2} \sum_{k=0}^{\infty} a_{k} x^{k \alpha}=0 .
\end{gathered}
$$

In the last equation, substitute $k+2$ for $k$ in the first term to get:

$$
\begin{gathered}
\sum_{k=0}^{\infty} \alpha^{2}(k+2)(k+1) a_{k+2} x^{k \alpha}-\sum_{k=2}^{\infty} \alpha^{2} k(k-1) a_{k} x^{k \alpha}-\sum_{k=1}^{\infty} \alpha^{2} k a_{k} x^{k \alpha}+ \\
\sum_{k=0}^{\infty} \alpha^{2} n^{2} a_{k} x^{k \alpha}=0 .
\end{gathered}
$$

Rewriting the last equation so that the summations start counting with $k=2$ yields:

$$
\begin{gathered}
\left(2 \alpha^{2} a_{2}+\alpha^{2} n^{2} a_{0}\right)+\left[6 \alpha^{2} a_{3}-\alpha^{2} a_{1}+\alpha^{2} n^{2} a_{1}\right] x^{\alpha} \\
+\sum_{k=2}^{\infty}\left[\alpha^{2}(k+2)(k+1) a_{k+2}-\alpha^{2} k(k-1) a_{k}-\alpha^{2} k a_{k}+\alpha^{2} n^{2} a_{k}\right] x^{k \alpha}=0 .
\end{gathered}
$$

To compute the values of the parameters of the fractional power series, the coefficients on both sides are compared. The constant coefficient yields

$$
2 \alpha^{2} a_{2}+\alpha^{2} n^{2} a_{0}=0 .
$$


Solving for $a_{2}$ gives

$$
a_{2}=\frac{-n^{2}}{2} a_{0}
$$

The coefficient of $x^{\alpha}$ yields

$$
6 \alpha^{2} a_{3}-\alpha^{2} a_{1}+\alpha^{2} n^{2} a_{1}=0 .
$$

Solving for $a_{3}$ gives

$$
a_{3}=-\frac{n^{2}-1}{6} a_{1}
$$

The general term for the coefficient of $x^{k \alpha}$ yields

$$
\alpha^{2}(k+2)(k+1) a_{k+2}-\alpha^{2} k(k-1) a_{k}-\alpha^{2} k a_{k}+\alpha^{2} n^{2} a_{k}=0 .
$$

This is solved for $a_{k+2}$ to yield:

$$
a_{k+2}=\frac{k^{2}-n^{2}}{(k+2)(k+1)} a_{k}
$$

This can be rewritten in the form

$$
a_{k+2}=-\frac{(n-k)(n+k)}{(k+2)(k+1)} a_{k}
$$

The fractional power series contains either even or odd terms. Consequently, there are two independent solutions. The first solution is the even-terms solution, and the second solution is the odd-terms solution. Both solutions diverge at $x= \pm 1$. The only interesting solutions are polynomial solutions. For the even terms, substituting $k=0$ in the last formula gives (7) for $a_{2}$. Substituting $k=2,4,6, \ldots$ in the last formula gives $a_{4}, a_{6}, a_{8}, \ldots$ as follows:

$$
\begin{gathered}
a_{4}=\frac{(n-2)(n+2)}{4(3)} \frac{n^{2}}{2} a_{0}, \\
a_{6}=-\frac{(n-4)(n+4)}{6(5)} \frac{(n-2)(n+2)}{4(3)} \frac{n^{2}}{2} a_{0}, \\
a_{8}=\frac{(n-6)(n+6)}{8(7)} \frac{(n-4)(n+4)}{6(5)} \frac{(n-2)(n+2)}{4(3)} \frac{n^{2}}{2} a_{0} .
\end{gathered}
$$

The general term looks as follows:

$$
a_{2 k}=(-1)^{k} \frac{(n-2(k-1))(n+2(k-1))}{2 k(2 k-1)} \cdots \frac{(n-4)(n+4)}{6(5)} \frac{(n-2)(n+2)}{4(3)} \frac{n(n)}{2(1)} a_{0} .
$$

This can be written as follows:

$$
a_{2 k}=\frac{(-1)^{k}}{(2 k) !} \prod_{i=0}^{k-1}(n-2 i)(n+2 i) a_{0}
$$

where $\prod$ is the product symbol. If we set

$$
a_{0}=(-1)^{\left[\frac{n}{2}\right]}
$$

gives the following conformable Chebyshev polynomials:

$$
\begin{gathered}
T_{0}(x)=1, \\
T_{2}(x)=2 x^{2 \alpha}-1,
\end{gathered}
$$




$$
\begin{gathered}
T_{4}(x)=8 x^{4 \alpha}-8 x^{2 \alpha}+1, \\
T_{6}(x)=32 x^{6 \alpha}-48 x^{4 \alpha}+18 x^{2 \alpha}-1, \\
T_{8}(x)=128 x^{8 \alpha}-256 x^{6 \alpha}+160 x^{4 \alpha}-32 x^{2 \alpha}+1 .
\end{gathered}
$$

Similarly, when the fractional power series contains only odd terms, then the odd terms are given in the following compact form:

$$
a_{2 k+1}=\frac{(-1)^{k}}{(2 k+1) !} \prod_{i=0}^{k-1}(n-1-2 i)(n+1+2 i) a_{1} .
$$

If we set

$$
a_{1}=(-1)^{\left[\frac{n}{2}\right]} n
$$

gives the following conformable Chebyshev polynomials:

$$
\begin{gathered}
T_{1}(x)=x^{\alpha}, \\
T_{3}(x)=4 x^{3 \alpha}-3 x^{\alpha}, \\
T_{5}(x)=16 x^{5 \alpha}-20 x^{3 \alpha}+5 x^{\alpha}, \\
T_{7}(x)=64 x^{7 \alpha}-112 x^{5 \alpha}+56 x^{3 \alpha}-7 x^{\alpha} .
\end{gathered}
$$

To derive a Rodrigues' type formula for the Chebyshev-I polynomials, $T_{n}(x)$, a polynomial $t_{n}(x)$ is to be necessitated that fulfills the Chebyshev orthogonality:

$$
\int_{-1}^{1} \frac{t_{n}(x) p_{n-1}(x)}{\sqrt{1-x^{2}}} d x=0, \forall p_{n-1} \in \mathcal{P}_{n-1} .
$$

Rewrite the integrand in (12) in the form

$$
\frac{t_{n}(x)}{\sqrt{1-x^{2}}} p_{n-1}(x)=\left(\frac{d^{n}}{d x^{n}} \phi_{n}(x)\right) p_{n-1}(x)
$$

and integrate the orthogonality conditions by parts $n$ times to get

$$
\left.0=\int_{-1}^{1}\left(\frac{d^{n}}{d x^{n}} \phi_{n}(x)\right) p_{n-1}(x) d x=\sum_{i=0}^{n-1}(-1)^{i} \phi_{n}^{(n-1-i)}(x) p_{n-1}^{(i)}(x)\right]_{x=-1}^{1}
$$

The last formula is valid for any polynomial of degree $\leq n-1$, thus we acquire the following conditions on $\phi_{n}(x)$ and its derivatives at $x=-1,1$ as follows

$$
\begin{array}{r}
\phi_{n}(-1)=\phi_{n}^{\prime}(-1)=\cdots=\phi_{n}^{(n-1)}(-1)=0, \\
\phi_{n}(1)=\phi_{n}^{\prime}(1)=\cdots=\phi_{n}^{(n-1)}(1)=0 .
\end{array}
$$

The polynomial $t_{n}(x)$ is of degree $n$ and thus

$$
0=\frac{d^{n+1} t_{n}(x)}{d x^{n+1}}=\frac{d^{n+1}}{d x^{n+1}}\left(\sqrt{1-x^{2}} \frac{d^{n} \phi_{n}(x)}{d x^{n}}\right) .
$$

The (15) with the conditions in (14) form a differential equation with $2 n$ boundary conditions and has the solution

$$
\phi_{n}(x)=b_{n}\left(1-x^{2}\right)^{n-\frac{1}{2}}, \text { for some constant } b_{n} .
$$

Consequently, the polynomial $t_{n}(x)$ has the form 


$$
t_{n}(x)=b_{n} \sqrt{1-x^{2}} \frac{d^{n}}{d x^{n}}\left[\left(1-x^{2}\right)^{n-\frac{1}{2}}\right] .
$$

Normalizing

$$
t_{n}(1)=T_{n}(1)
$$

yields

$$
(-1)^{n}(2 n-1)(2 n-3) \cdots(5)(3)(1) b_{n}=1 \text {. }
$$

And thus

$$
b_{n}=\frac{(-1)^{n}}{(2 n-1) ! !}
$$

where $(2 n-1) ! !=(2 n-1)(2 n-3)(\cdots(3)(1)$ denotes the double factorial. Since

$$
(2 n-1) ! !=\frac{(2 n) !}{n ! 2^{n}}
$$

thus

$$
b_{n}=\frac{(-2)^{n} n !}{(2 n) !}
$$

Thus, the Chebyshev-I polynomials are given by the following Rodrigues' type formula:

$$
T_{n}(x)=\frac{(-2)^{n} n !}{(2 n) !}\left(1-x^{2}\right)^{\frac{1}{2}} \frac{d^{n}}{d x^{n}}\left[\left(1-x^{2}\right)^{n-\frac{1}{2}}\right] .
$$

The conformable Chebyshev-I polynomials can be expressed by the following Rodrigues' type formula:

$$
T_{n}(x)=\frac{(-2)^{n} n !}{(2 n) ! \alpha^{n}}\left(1-x^{2 \alpha}\right)^{\frac{1}{2}} D^{n \alpha}\left[\left(1-x^{2 \alpha}\right)^{n-\frac{1}{2}}\right], n \in N, \alpha \in(0,1] .
$$

It is also interesting to discuss and find a similar generalization to orthogonal polynomials on triangular domains [14].

\section{CONCLUSION}

In this paper, the conformable Chebyshev differential equation of the first kind is introduced in formula (5), using the conformable derivative defined in formula (2). Then the explicit form of the conformable Chebyshev functions are derived in the form of a power series and a Ridrigue's type formula is also derived in (20). We consider the conformable derivative given in formula (2) of a function $f(x)$. This means that the ordinary derivative $f^{\prime}(x)$ exists and $f^{\prime}(x)=D^{1} f(x)$. Moreover, $D^{\alpha} f(x)=x^{1-\alpha} f^{\prime}(x)$ and $D^{\alpha} D^{\alpha} f(x)=(1-\alpha) x^{1-2 \alpha} f^{\prime}(x)+x^{2-2 \alpha} f^{\prime \prime}(x)$ for $\alpha \in(0,1]$. From these formulas, it is clear that, if the ordinary Chebyshev polynomials $y(x)=T_{n}(x)$ are solutions of the ordinary Chebyshev differential equation, then the functions $u(x)=y\left(x^{\alpha}\right)=T_{n}\left(x^{\alpha}\right)$ are solutions of the conformable differential (5).

\section{ACKNOWLEDGEMENT}

The author owes thanks for the reviewers for invaluable suggestions to improve an earlier version of this paper. This research was funded by Jordan University of Science and Technology.

\section{REFERENCES}

[1] Baleanu, D., Diethelm, K., Scalas, E. and Trujillo, J., "Fractional Calculus: Models And Numerical Methods," Fractional Calculus: Models And Numerical Methods, 2016. 
[2] Baleanu, D. and , Mustafa, O, "Asymptotic Integration and Stability: For Ordinary, Functional and Discrete Differential Equations of Fractional Order," Asymptotic Integration and Stability: For Ordinary, Functional and Discrete Differential Equations of Fractional Order, 2015.

[3] Khalil R., Al Horani M., Yousef A., and Sababheh M, "A new definition of fractional derivative," Journal of Computational Applied Mathematics, vol. 264, pp. 65-70, 2014.

[4] Abdeljawad T., "On conformable fractional calculus," Journal of Computational Applied Mathematics, vol. 279, pp. 57-66, 2015.

[5] SET, E., CHOI, J. and DEMIRBAS, S., "Some New Chebyshev Type Inequalities Forfractional Integral Operator Containing A Furtherextension Of Mittag-Leffler Function In The Kernel," 2019.

[6] Mason J. and Handscomb, D. "Chebyshev Polynomials," Chebyshev Polynomials,2003.

[7] Szegö., "G. Orthogonal Polynomials," Orthogonal Polynomials, 1975.

[8] Rababah., et al., "Computing Derivatives of Jacobi Polynomials using Bernstein Transformation and Differentiation Matrix," Numerical Functional Analysis and Optimization, vol. 29, no. 5-6, pp. 660-673, 2008.

[9] Rababah, A., ,'Transformation of Chebyshev-Bernstein polynomial basis," Comput. Meth. Appl. Math., vol. 3, no. 4, pp. 608-622, 2003.

[10] Rababah, A., "Jacobi-Bernstein basis transfoprmation," Comput. Meth. Appl. Math., vol. 4, no. 2, pp. 206-214, 2004.

[11] Rababah, A., "Integration of Jacobi and Weighted Bernstein Polynomials Using Bases Transformations," Comput. Meth. Appl. Math., vol. 7, no. 3, pp. 221-226, 2007.

[12] Y. Simsek and G. Ozdemir, "Some Identities Of The Humbert And Generalized Chebyshev Polynomials", International Conference on Analysis and Its Applications (ICAA-2016), pp. 124-124, 2016.

[13] Y. Simsek and M. Gunay, "On Bernstein type polynomials and their Applications," Advances in Difference Equations, vol. 79, 2015.

[14] Rababah A. and Alqudah M., "Jacobi-weighted orthogonal polynomials on triangular domains," Journal of Applied Mathematics, pp. 205-217, 2005.

[15] A. Rababah, "L-2 degree reduction of triangular Bèzier surfaces with common tangent planes at vertices," International Journal of Computational Geometry and Applications, vol. 15, no. 5, pp. 477-490, 2005.

[16] A. Rababah, "Distances with rational triangular Bèzier surfaces," Applied Mathematics and Computation, vol. 160, pp. 379-386, 2015.

[17] A. Rababah., et al., "Multiple Degree Reduction and Elevation of Bézier Curves Using Jacobi-Bernstein Basis Transformations," Numerical Functional Analysis and Optimization, vol. 28, no. 9-10, pp. 11791196, 2007.

[18] A. Rababah, "Approximation von Kurven mit Polynomen und Splines," Ph. Dissertation, Stuttgart Universität, West Germany, 1992.

[19] A. Rababah, "Taylor theorem for planar curves," Proceedings of the American Mathematical Society, vol. 119, no. 3, pp. 803-810, 1993.

[20] A. Rababah, "The best uniform quadratic approximation of circular arcs with high accuracy," Open Mathematics, vol. 14, no. 1, pp. 118-127, 2016.

[21] A. Rababah, "The best quintic Chebyshev approximation of circular arcs of order ten," International Journal of Electrical and Computer Engineering (IJECE), vol. 9, no. 5, pp. 3779-3785, 2019.

[22] Ashwak ALabaichi, Maisa'a Abid Ali K. Al-Dabbas, and Adnan Salih, "Image steganography using least significant bit and secret map techniques," International Journal of Electrical and Computer Engineering (IJECE), vol. 10, no. 1, pp. 935-946, 2020.

[23] Shima Ramesh Maniyath and Thanikaiselvan V., "Novel efficient multiple encryption algorithmfor real time images," International Journal of Electrical and Computer Engineering (IJECE), vol. 10, no. 2, pp. 1327-1336, 2020.

[24] R. Rizal Isnanto, Munawar Agus Riyadi, and Muhammad Fahmi Awaj, "Herb Leaves Recognition using Gray Level Co-occurrence Matrix and Five Distance-based Similarity Measures," International Journal of Electrical and Computer Engineering (IJECE), vol. 8, no. 3, pp. 1920-1932, 2018.

[25] A. Rababah and M. Jaradat, "Approximating offset curves using B ézier curves with high accuracy," International Journal of Electrical and Computer Engineering, vol. 10, no. 2, pp. 1648-1654, 2020.

[26] A. Rababah and E. Rababah, The best sextic approximation of hyperbola with order twelve," International Journal of Electrical and Computer Engineering (IJECE), vol. 10, no. 2, pp. 2192-2199, 2020. 


\section{BIOGRAPHIES OF AUTHORS}

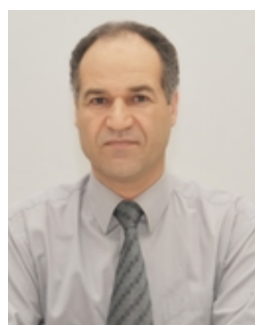

Abedallah Rababah is a professor of mathematics at United Arab Emirates University and is on leave from Jordan University of Science and Technology. He is working in the field of Computer Aided Geometric Design, abbreviated CAGD. In particular, his research is on degree raising and reduction of Bézier curves and surfaces with geometric boundary conditions, Bernstein polynomials, and their duality. He is known for his research in describing approximation methods that significantly improve the standard rates obtained by classical (local Taylor, Hermite) methods. He proved the following conjecture for a particular set of curves of nonzero measure: Conjecture: A smooth regular planar curve can, in general, be approximated by a polynomial curve of degree $n$ with order $2 \mathrm{n}$. The method exploited the freedom in the choice of the parametrization and achieved the order $4 \mathrm{n} / 3$, rather than $n+1$. Generalizations were also proved for space curves. Professor Rababah is also doing research in the fields of classical approximation theory, orthogonal polynomials, Jacobi-weighted orthogonal polynomials on triangular domains, and best uniform approximations. Since 1992, He has been teaching at German, Jordanian, American, Canadian, and Emirates' universities. He is active in the editorial boards of many journals in mathematics and computer science. Further info can be found on his homepage at ResearchGate:

https://www.researchgate.net/profile/Abedallah_Rababah

or at http://www.just.edu.jo/eportfolio/Pages/Default.aspx?email=rababah 